Supporting informations for:

\title{
A DFT Study of Stannane Dehydrocoupling Catalyzed by $\mathrm{Cp}_{2} \mathrm{LaH}$
}

Christophe Raynaud ${ }^{\mathrm{a}}$, Lionel Perrin ${ }^{\mathrm{a}, \mathrm{b}}$ and Laurent Maron $^{\mathrm{a},{ }^{*}}$

a: Laboratoire de Physique Quantique - IRSAMC - Université Paul Sabatier and CNRS

(UMR 5626) - 118 route de Narbonne - 31064 Toulouse Cedex, France

b : Protéines Membranaires Transductrices d'Energie, URA 2096 du CNRS DSV/DBJC/SBFM - CEA-Saclay - 91191 Gif-sur-Yvette Cedex, France

* to whom correspondence should be addressed. E-mail: Laurent.Maron@irsamc.ups-tlse.fr 
Thermodynamic data (absolute electronic energies, ZPE corrections, enthalpies and free energies in atomic units. See the text for the correspondence between labels and structures).

\begin{tabular}{|c|c|c|c|c|}
\hline Name/Number & $\mathrm{E}_{\mathrm{p}}$ (a.u.) & $\begin{array}{l}\text { ZPE corr. } \\
\text { (a.u.) }\end{array}$ & $\mathrm{H}^{\circ}$ (a.u.) & $\mathrm{G}^{\circ}$ (a.u.) \\
\hline $\mathrm{Cp}_{2} \mathrm{LaH}$ & -419.205466 & 0.170848 & -419.022442 & -419.076103 \\
\hline $\mathrm{H}_{2}$ & -1.177517 & 0.010150 & -1.164062 & -1.178856 \\
\hline $\mathrm{SnH}_{4}$ & -5.767037 & 0.025564 & -5.737179 & -5.765436 \\
\hline $\begin{array}{c}\mathrm{Sn}_{2} \mathrm{H}_{6} \\
\mathrm{SnH}_{2} \\
(\text { sinolet) }\end{array}$ & -10.374753 & 0.039537 & -10.328050 & -10.366878 \\
\hline (singlet) & -4.554036 & 0.009533 & -4.540644 & -4.567360 \\
\hline 3 & -424.977838 & 0.197832 & -424.763172 & -424.828469 \\
\hline 2 & -424.977009 & 0.196807 & -424.764245 & -424.826896 \\
\hline 4 & -425.010185 & 0.199348 & -424.793705 & -424.858170 \\
\hline 5 & -423.828766 & 0.185022 & -423.628272 & -423.691910 \\
\hline $5^{\prime}$ & -423.821959 & 0.185232 & -423.620880 & -423.684823 \\
\hline 7 & -429.604410 & 0.209205 & -429.376694 & -429.445804 \\
\hline 6 & -429.593502 & 0.212265 & -429.362455 & -429.432577 \\
\hline 8 & -429.592892 & 0.209989 & -429.364323 & -429.433692 \\
\hline 9 & -429.592574 & 0.209989 & -429.364005 & -429.433374 \\
\hline 10 & -429.596595 & 0.212019 & -429.364813 & -429.438804 \\
\hline 12 & -423.779202 & 0.182239 & -423.581774 & -423.642320 \\
\hline 13 & -423.787386 & 0.183541 & -423.588261 & -423.648921 \\
\hline 16 & -10.334026 & 0.038542 & -10.288279 & -10.327635 \\
\hline 15 & -10.321599 & 0.037139 & -10.277304 & -10.315927 \\
\hline 18 & -429.604410 & 0.209205 & -429.376694 & -429.445804 \\
\hline 17 & -429.545544 & 0.209595 & -429.315882 & -429.391491 \\
\hline
\end{tabular}


Cartesian coordinates of all optimized structures (Angstroms). See the text for the correspondence between structures and labels.

\section{$\mathbf{H}_{2}$}

$\begin{array}{llll}\mathrm{H} & 0.000000 & 0.000000 & -0.011702\end{array}$

$\mathrm{H} \quad 0.000000 \quad 0.000000 \quad 0.731702$

\begin{tabular}{cccc}
\multicolumn{2}{c}{ SnH $_{2}$ (singlet) } & & \\
Sn & -0.137903 & 0.000000 & -0.079999 \\
H & 0.322358 & 0.000000 & 1.649606 \\
H & 1.589338 & 0.000000 & -0.545507
\end{tabular}

\section{$\mathrm{SnH}_{4}$}

$\begin{array}{crrc}\text { Sn } & 0.000020 & 0.000000 & -0.000405 \\ \mathrm{H} & 0.000033 & 0.000000 & 1.716863 \\ \mathrm{H} & 1.619099 & 0.000000 & -0.572761 \\ \mathrm{H} & -0.809576 & -1.402121 & -0.572815 \\ \mathrm{H} & -0.809576 & 1.402121 & -0.572815\end{array}$

\section{$\mathrm{Sn}_{2} \mathrm{H}_{6}$}

$\begin{array}{rrrr}\text { Sn } & 0.000001 & 0.000074 & 0.217435 \\ \mathrm{Sn} & 0.000001 & -0.000073 & 3.036165 \\ \mathrm{H} & 1.394346 & 0.805252 & -0.389096 \\ \mathrm{H} & 0.000005 & -1.609993 & -0.389213 \\ \mathrm{H} & -1.394353 & 0.805246 & -0.389090 \\ \mathrm{H} & -1.394346 & -0.805260 & 3.642690 \\ \mathrm{H} & 1.394356 & -0.805238 & 3.642697 \\ \mathrm{H} & -0.000012 & 1.609992 & 3.642813\end{array}$

\section{$\mathrm{Cp}_{2} \mathrm{LaH}$}

$\begin{array}{crrc}\mathrm{La} & -0.471739 & -0.491618 & -0.000088 \\ \mathrm{H} & -2.317395 & 0.594614 & -0.001180 \\ \mathrm{C} & 1.524463 & -0.030366 & 1.929198 \\ \mathrm{C} & 0.673263 & 1.105200 & 1.990733 \\ \mathrm{C} & -0.559099 & 0.703245 & 2.565252 \\ \mathrm{C} & -0.476158 & -0.683024 & 2.842148 \\ \mathrm{C} & 0.812715 & -1.135842 & 2.454761 \\ \mathrm{C} & 1.525762 & -0.030743 & -1.928114 \\ \mathrm{C} & 0.815185 & -1.137175 & -2.453250 \\ \mathrm{C} & -0.473668 & -0.685565 & -2.842132 \\ \mathrm{C} & -0.557756 & 0.700899 & -2.566577 \\ \mathrm{C} & 0.673856 & 1.104198 & -1.991392 \\ \mathrm{H} & 2.551670 & -0.039185 & 1.583857 \\ \mathrm{H} & 0.938467 & 2.114848 & 1.698462 \\ \mathrm{H} & -1.410024 & 1.342530 & 2.759897 \\ \mathrm{H} & -1.247213 & -1.281794 & 3.315145\end{array}$




$\begin{array}{crrr}\mathrm{H} & 1.203886 & -2.139471 & 2.585828 \\ \mathrm{H} & 2.552694 & -0.038554 & -1.581938 \\ \mathrm{H} & 1.207132 & -2.140662 & -2.583069 \\ \mathrm{H} & -1.243920 & -1.285278 & -3.315246 \\ \mathrm{H} & -1.408933 & 1.339458 & -2.762501 \\ \mathrm{H} & 0.938134 & 2.114284 & -1.699794\end{array}$

\section{3}

$\begin{array}{llll}\mathrm{La} & 0.103964 & -0.457323 & -0.016276\end{array}$

$\begin{array}{llll}\text { Sn } & -3.248654 & -1.809648 & 0.043967\end{array}$

$\begin{array}{llll}\mathrm{H} & -1.697273 & -2.600797 & 0.038238\end{array}$

$\begin{array}{llll}\mathrm{H} & 0.634838 & -2.539865 & 0.007432\end{array}$

$\begin{array}{llll}\mathrm{H} & -4.123958 & -2.113349 & 1.477254\end{array}$

$\begin{array}{llll}\mathrm{H} & -2.793872 & -0.121502 & 0.007795\end{array}$

$\begin{array}{llll}\mathrm{H} & -4.159324 & -2.164137 & -1.355272\end{array}$

$\begin{array}{llll}\text { C } & 1.938903 & 0.162956 & 2.019993\end{array}$

$\begin{array}{llll}\text { C } & 1.121835 & 1.321855 & 1.926191\end{array}$

$\begin{array}{llll}\text { C } & -0.160521 & 0.991280 & 2.425068\end{array}$

$\begin{array}{llll}\text { C } & -0.136491 & -0.370829 & 2.826518\end{array}$

$\begin{array}{llll}\text { C } & 1.163948 & -0.879591 & 2.585079\end{array}$

$\begin{array}{llll}\text { C } & 1.914949 & 0.114336 & -2.087597\end{array}$

$\begin{array}{llll}\text { C } & 1.135956 & -0.943840 & -2.617199\end{array}$

$\begin{array}{llll}\text { C } & -0.168076 & -0.443795 & -2.857333\end{array}$

$\begin{array}{llll}\text { C } & -0.190576 & 0.928082 & -2.490658\end{array}$

$\begin{array}{llll}\text { C } & 1.096499 & 1.273714 & -2.014524\end{array}$

$\begin{array}{llll}\mathrm{H} & 2.987747 & 0.101062 & 1.752663\end{array}$

$\begin{array}{llll}\mathrm{H} & 1.438697 & 2.298573 & 1.579090\end{array}$

$\begin{array}{llll}\mathrm{H} & -0.998887 & 1.672211 & 2.525675\end{array}$

$\begin{array}{llll}\mathrm{H} & -0.951158 & -0.910986 & 3.296736\end{array}$

$\begin{array}{llll}\mathrm{H} & 1.506648 & -1.883260 & 2.799438\end{array}$

$\begin{array}{llll}\mathrm{H} & 2.966852 & 0.061329 & -1.830513\end{array}$

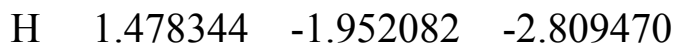

$\begin{array}{llll}\mathrm{H} & -0.986924 & -0.997529 & -3.303887\end{array}$

$\begin{array}{llll}\mathrm{H} & -1.031257 & 1.604848 & -2.599814\end{array}$

H $\quad 1.414925 \quad 2.259605 \quad-1.696071$

$\begin{array}{lrrl}\mathbf{2} & & & \\ \mathrm{La} & 0.002452 & -0.361612 & -0.015974 \\ \mathrm{Sn} & -3.057055 & -1.918272 & 0.049945 \\ \mathrm{H} & -1.315017 & -2.497079 & 0.061683 \\ \mathrm{H} & 0.283739 & -2.508886 & 0.054681 \\ \mathrm{H} & -3.934013 & -2.288086 & 1.474413 \\ \mathrm{H} & -2.889296 & -0.160714 & -0.012393 \\ \mathrm{H} & -3.946301 & -2.386345 & -1.337594 \\ \mathrm{C} & 1.865839 & 0.145195 & 2.027253 \\ \mathrm{C} & 1.111758 & 1.346832 & 1.936648 \\ \mathrm{C} & -0.188740 & 1.082476 & 2.428879 \\ \mathrm{C} & -0.240136 & -0.280771 & 2.822233\end{array}$




$\begin{array}{rrrr}\mathrm{C} & 1.032982 & -0.857047 & 2.582316 \\ \mathrm{C} & 1.869420 & 0.006395 & -2.086320 \\ \mathrm{C} & 1.026373 & -1.018860 & -2.580861 \\ \mathrm{C} & -0.241470 & -0.445553 & -2.853630 \\ \mathrm{C} & -0.176524 & 0.938070 & -2.541627 \\ \mathrm{C} & 1.127074 & 1.218409 & -2.066675 \\ \mathrm{H} & 2.911676 & 0.029678 & 1.765994 \\ \mathrm{H} & 1.482512 & 2.307368 & 1.597572 \\ \mathrm{H} & -0.990607 & 1.805947 & 2.529237 \\ \mathrm{H} & -1.084623 & -0.777880 & 3.286598 \\ \mathrm{H} & 1.321328 & -1.877725 & 2.797810 \\ \mathrm{H} & 2.914343 & -0.103719 & -1.819073 \\ \mathrm{H} & 1.304932 & -2.053143 & -2.735944 \\ \mathrm{H} & -1.090998 & -0.961064 & -3.287832 \\ \mathrm{H} & -0.971175 & 1.662333 & -2.684706 \\ \mathrm{H} & 1.507047 & 2.193660 & -1.784505\end{array}$

$\begin{array}{rrrr}\mathbf{4} & & & \\ \mathrm{La} & -0.115407 & -0.423422 & -0.009948 \\ \mathrm{Sn} & -3.205170 & -1.346933 & 0.023605 \\ \mathrm{H} & -0.385056 & -3.074513 & 0.054765 \\ \mathrm{H} & 0.374767 & -3.081068 & 0.053138 \\ \mathrm{H} & -4.306882 & -1.078020 & 1.357068 \\ \mathrm{H} & -2.412210 & 0.371938 & -0.030530 \\ \mathrm{H} & -4.309741 & -1.161300 & -1.321784 \\ \mathrm{C} & 1.818906 & 0.248127 & 1.918942 \\ \mathrm{C} & 0.868917 & 1.301998 & 1.976261 \\ \mathrm{C} & -0.324195 & 0.785733 & 2.543264 \\ \mathrm{C} & -0.117803 & -0.587078 & 2.827925 \\ \mathrm{C} & 1.208489 & -0.918503 & 2.443771 \\ \mathrm{C} & 1.816115 & 0.138815 & -1.976916 \\ \mathrm{C} & 1.211127 & -1.060515 & -2.429073 \\ \mathrm{C} & -0.116887 & -0.759357 & -2.832166 \\ \mathrm{C} & -0.329756 & 0.627170 & -2.631803 \\ \mathrm{C} & 0.860880 & 1.182503 & -2.097296 \\ \mathrm{H} & 2.844863 & 0.334094 & 1.579834 \\ \mathrm{H} & 1.044522 & 2.332763 & 1.689753 \\ \mathrm{H} & -1.228108 & 1.347198 & 2.747257 \\ \mathrm{H} & -0.831499 & -1.252207 & 3.299263 \\ \mathrm{H} & 1.688543 & -1.881334 & 2.580000 \\ \mathrm{H} & 2.841594 & 0.250045 & -1.643799 \\ \mathrm{H} & 1.695763 & -2.027577 & -2.506549 \\ \mathrm{H} & -0.827528 & -1.455697 & -3.261217 \\ \mathrm{H} & -1.236431 & 1.170782 & -2.869138 \\ \mathrm{H} & 1.031666 & 2.229690 & -1.874477\end{array}$

\section{5}

$\begin{array}{llll}\text { C } & 1.929592 & 0.718440 & -2.033848\end{array}$ 


$\begin{array}{cccc}\mathrm{C} & 2.405458 & 1.198857 & -0.787816 \\ \mathrm{C} & 2.831699 & 0.083875 & -0.019122 \\ \mathrm{C} & 2.616018 & -1.085470 & -0.790194 \\ \mathrm{C} & 2.053256 & -0.695712 & -2.032801 \\ \mathrm{La} & 0.008281 & -0.163229 & -0.176592 \\ \mathrm{C} & -2.185603 & -0.610968 & -1.881756 \\ \mathrm{C} & -2.704560 & -0.874302 & -0.587824 \\ \mathrm{C} & -2.766874 & 0.352372 & 0.118567 \\ \mathrm{C} & -2.288933 & 1.376766 & -0.740752 \\ \mathrm{C} & -1.935630 & 0.782752 & -1.978792 \\ \mathrm{Sn} & 0.050594 & -1.382492 & 2.810450 \\ \mathrm{H} & 3.281584 & 0.121415 & 0.965699 \\ \mathrm{H} & 2.865003 & -2.096892 & -0.491462 \\ \mathrm{H} & 1.815762 & -1.355114 & -2.860277 \\ \mathrm{H} & 1.575975 & 1.325952 & -2.858810 \\ \mathrm{H} & 2.482447 & 2.240694 & -0.495332 \\ \mathrm{H} & -3.151556 & 0.487854 & 1.122259 \\ \mathrm{H} & -2.254422 & 2.436475 & -0.511096 \\ \mathrm{H} & -1.578942 & 1.305758 & -2.858433 \\ \mathrm{H} & -2.058499 & -1.336773 & -2.677362 \\ \mathrm{H} & -3.024372 & -1.839204 & -0.212634 \\ \mathrm{H} & -1.292673 & -2.229688 & 3.550990 \\ \mathrm{H} & -0.028717 & -2.232124 & 1.106920 \\ \mathrm{H} & 1.378757 & -2.316516 & 3.469746\end{array}$

5'

$\begin{array}{llll}\text { C } & -2.538460 & -0.787764 & -0.478691\end{array}$

$\begin{array}{llll}\text { C } & -2.793843 & 0.451267 & 0.157522\end{array}$

$\begin{array}{llll}\text { C } & -2.399368 & 1.489758 & -0.725188\end{array}$

$\begin{array}{llll}\text { C } & -1.892917 & 0.893350 & -1.907087\end{array}$

$\begin{array}{llll}\text { C } & -1.972956 & -0.516137 & -1.751949\end{array}$

$\begin{array}{llll}\mathrm{La} & 0.041951 & 0.255013 & 0.007724\end{array}$

$\begin{array}{llll}\text { C } & 2.475414 & 1.264189 & -1.019273\end{array}$

$\begin{array}{llll}\text { C } & 2.882749 & 0.321929 & -0.038648\end{array}$

$\begin{array}{llll}\text { C } & 2.538118 & -0.971739 & -0.500323\end{array}$

$\begin{array}{llll}\text { C } & 1.903175 & -0.828766 & -1.761793\end{array}$

C $\quad 1.872114 \quad 0.553303 \quad-2.086129$

$\begin{array}{llll}\mathrm{Sn} & -0.025958 & -2.220465 & 2.226365\end{array}$

$\begin{array}{llll}\mathrm{H} & 3.406502 & 0.544983 & 0.884357\end{array}$

$\mathrm{H} \quad 2.751196 \quad-1.904936 \quad 0.005181$

H $\quad 1.560129 \quad-1.638395 \quad-2.395693$

$\begin{array}{llll}\mathrm{H} & 1.495898 & 0.983076 & -3.006918\end{array}$

$\begin{array}{llll}\mathrm{H} & 2.644236 & 2.335492 & -0.984708\end{array}$

$\mathrm{H} \quad-3.249273 \quad 0.578815 \quad 1.133130$

$\mathrm{H} \quad-2.515429 \quad 2.554376 \quad-0.549921$

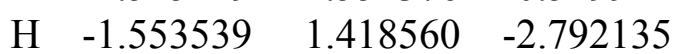

$\mathrm{H} \quad-1.706972 \quad-1.254035 \quad-2.499928$

$\mathrm{H} \quad-2.760536 \quad-1.767687 \quad-0.075749$

$\mathrm{H} \quad-1.296610 \quad-2.196026 \quad 3.434989$ 


$$
\begin{array}{lrrr}
\mathrm{H} & -0.254492 & -3.809839 & 1.523687 \\
\mathrm{H} & 1.394955 & -2.483909 & 3.218873
\end{array}
$$

$\begin{array}{cccc}7 & & & \\ \mathrm{La} & -0.180140 & -0.559449 & -0.310413 \\ \mathrm{C} & 2.653032 & -0.813428 & -0.282825 \\ \mathrm{C} & 2.226729 & -1.549012 & -1.417518 \\ \mathrm{C} & 1.720898 & -0.631209 & -2.372255 \\ \mathrm{C} & 1.841564 & 0.676214 & -1.830437 \\ \mathrm{C} & 2.420035 & 0.562043 & -0.541670 \\ \mathrm{C} & -2.224165 & -0.543011 & -2.233765 \\ \mathrm{C} & -2.790983 & -1.265996 & -1.153619 \\ \mathrm{C} & -3.012076 & -0.360267 & -0.086342 \\ \mathrm{C} & -2.592244 & 0.927394 & -0.511651 \\ \mathrm{C} & -2.105258 & 0.815615 & -1.837492 \\ \mathrm{H} & 3.116109 & -1.227901 & 0.604765 \\ \mathrm{H} & 2.298917 & -2.622984 & -1.540683 \\ \mathrm{H} & 1.356322 & -0.877701 & -3.362883 \\ \mathrm{H} & 1.586944 & 1.600490 & -2.336479 \\ \mathrm{H} & 2.681885 & 1.386484 & 0.111883 \\ \mathrm{H} & -1.978567 & -0.944953 & -3.210055 \\ \mathrm{H} & -3.033289 & -2.321918 & -1.150799 \\ \mathrm{H} & -3.467753 & -0.600977 & 0.866905 \\ \mathrm{H} & -2.675731 & 1.845557 & 0.058857 \\ \mathrm{H} & -1.751199 & 1.630740 & -2.458165 \\ \mathrm{H} & 1.413052 & 0.386165 & 3.883814 \\ \mathrm{Sn} & -0.002772 & 1.144405 & 3.310363 \\ \mathrm{Sn} & -0.207975 & -2.828098 & 2.005404 \\ \mathrm{H} & -0.049804 & 1.099538 & 1.554598 \\ \mathrm{H} & 0.080210 & 2.840111 & 3.603935 \\ \mathrm{H} & -1.450176 & 0.514026 & 3.955348 \\ \mathrm{H} & -0.302100 & -2.952027 & 0.127670 \\ \mathrm{H} & -1.579291 & -3.848478 & 2.388432 \\ \mathrm{H} & 1.095450 & -3.969987 & 2.263429\end{array}$

$\begin{array}{cccc}\mathbf{6} & & & \\ \mathrm{La} & -0.176406 & -0.501643 & -0.386675 \\ \mathrm{C} & 2.646155 & -0.842906 & -0.304871 \\ \mathrm{C} & 2.226539 & -1.568919 & -1.449585 \\ \mathrm{C} & 1.767389 & -0.639317 & -2.414825 \\ \mathrm{C} & 1.908916 & 0.665078 & -1.868241 \\ \mathrm{C} & 2.456447 & 0.538138 & -0.567890 \\ \mathrm{C} & -2.199909 & -0.394580 & -2.333036 \\ \mathrm{C} & -2.762774 & -1.204120 & -1.316230 \\ \mathrm{C} & -3.013787 & -0.381363 & -0.187802 \\ \mathrm{C} & -2.616028 & 0.941452 & -0.512233 \\ \mathrm{C} & -2.108547 & 0.933615 & -1.834646 \\ \mathrm{H} & 3.090569 & -1.265003 & 0.589093\end{array}$




$\begin{array}{crcc}\mathrm{H} & 2.280938 & -2.644192 & -1.576174 \\ \mathrm{H} & 1.418839 & -0.876368 & -3.413593 \\ \mathrm{H} & 1.685964 & 1.595553 & -2.377993 \\ \mathrm{H} & 2.714052 & 1.353486 & 0.097187 \\ \mathrm{H} & -1.936236 & -0.718324 & -3.333415 \\ \mathrm{H} & -2.993674 & -2.260087 & -1.397329 \\ \mathrm{H} & -3.485570 & -0.696043 & 0.735902 \\ \mathrm{H} & -2.712752 & 1.810118 & 0.127807 \\ \mathrm{H} & -1.760482 & 1.797990 & -2.388708 \\ \mathrm{H} & 1.680775 & 0.940494 & 3.335293 \\ \mathrm{Sn} & 0.016369 & 0.534277 & 3.175456 \\ \mathrm{Sn} & -0.346018 & -2.455630 & 2.204619 \\ \mathrm{H} & -0.060338 & 0.987401 & 1.303783 \\ \mathrm{H} & -1.057140 & 1.858261 & 3.476851 \\ \mathrm{H} & -0.319801 & -0.471527 & 4.581113 \\ \mathrm{H} & -0.365050 & -2.923098 & 0.434707 \\ \mathrm{H} & -1.813658 & -3.131726 & 2.817632 \\ \mathrm{H} & 0.922166 & -3.420904 & 2.874044\end{array}$

$\begin{array}{cccc}\mathbf{8} & & & \\ \mathrm{La} & -0.172793 & -0.488084 & -0.389784 \\ \mathrm{C} & 2.636435 & -0.934182 & -0.338429 \\ \mathrm{C} & 2.177242 & -1.638955 & -1.481563 \\ \mathrm{C} & 1.743114 & -0.688214 & -2.437369 \\ \mathrm{C} & 1.939012 & 0.607319 & -1.886098 \\ \mathrm{C} & 2.496242 & 0.454123 & -0.592929 \\ \mathrm{C} & -2.161918 & -0.269325 & -2.363205 \\ \mathrm{C} & -2.716241 & -1.178894 & -1.429675 \\ \mathrm{C} & -3.014314 & -0.461544 & -0.241598 \\ \mathrm{C} & -2.651793 & 0.894516 & -0.445085 \\ \mathrm{C} & -2.119749 & 1.013361 & -1.752319 \\ \mathrm{H} & 3.076317 & -1.377093 & 0.547751 \\ \mathrm{H} & 2.191297 & -2.714874 & -1.614194 \\ \mathrm{H} & 1.374578 & -0.907122 & -3.432989 \\ \mathrm{H} & 1.744505 & 1.548116 & -2.388513 \\ \mathrm{H} & 2.788043 & 1.256041 & 0.074081 \\ \mathrm{H} & -1.869158 & -0.499006 & -3.381324 \\ \mathrm{H} & -2.915091 & -2.230058 & -1.605351 \\ \mathrm{H} & -3.493561 & -0.866882 & 0.642351 \\ \mathrm{H} & -2.782392 & 1.701876 & 0.265057 \\ \mathrm{H} & -1.786056 & 1.931310 & -2.222840 \\ \mathrm{H} & 1.525106 & 1.286541 & 3.325524 \\ \mathrm{Sn} & -0.018105 & 0.535111 & 3.165538 \\ \mathrm{Sn} & -0.305461 & -2.379401 & 2.307044 \\ \mathrm{H} & 0.004484 & 1.030874 & 1.235596 \\ \mathrm{H} & -1.388704 & 1.561801 & 3.364514 \\ \mathrm{H} & -0.088371 & -0.419684 & 4.661243 \\ \mathrm{H} & -0.377434 & -2.873066 & 0.557871 \\ \mathrm{H} & -1.746507 & -3.037492 & 2.986902\end{array}$




\section{$\begin{array}{llll}\text { H } & 1.007309 & -3.296348 & 2.945787\end{array}$}

$\begin{array}{cccc}\text { 9 } & & & \\ \mathrm{C} & -2.084841 & 1.114966 & -1.752383 \\ \mathrm{C} & -2.124667 & -0.136925 & -2.422895 \\ \mathrm{C} & -2.693675 & -1.086044 & -1.538801 \\ \mathrm{C} & -3.005524 & -0.421822 & -0.323600 \\ \mathrm{C} & -2.636019 & 0.939588 & -0.458710 \\ \mathrm{La} & -0.161535 & -0.441521 & -0.429832 \\ \mathrm{C} & 2.491549 & 0.544146 & -0.576123 \\ \mathrm{C} & 2.655027 & -0.856233 & -0.431917 \\ \mathrm{C} & 2.197669 & -1.478257 & -1.622994 \\ \mathrm{C} & 1.744438 & -0.463217 & -2.500919 \\ \mathrm{C} & 1.922552 & 0.787390 & -1.851017 \\ \mathrm{Sn} & -0.249545 & -2.011224 & 2.668574 \\ \mathrm{Sn} & 0.029743 & 0.935090 & 3.405955 \\ \mathrm{H} & 3.101446 & -1.359498 & 0.418504 \\ \mathrm{H} & 2.232710 & -2.540141 & -1.842199 \\ \mathrm{H} & 1.373630 & -0.610271 & -3.508650 \\ \mathrm{H} & 1.708663 & 1.761207 & -2.276670 \\ \mathrm{H} & 2.766928 & 1.295687 & 0.152827 \\ \mathrm{H} & -1.819180 & -0.321052 & -3.446435 \\ \mathrm{H} & -2.898463 & -2.126432 & -1.767737 \\ \mathrm{H} & -3.490571 & -0.866272 & 0.538342 \\ \mathrm{H} & -2.765628 & 1.711998 & 0.288668 \\ \mathrm{H} & -1.740639 & 2.051923 & -2.175383 \\ \mathrm{H} & 1.592237 & 1.646427 & 3.301011 \\ \mathrm{H} & -0.022133 & 0.895877 & 1.273639 \\ \mathrm{H} & -1.379772 & 1.920151 & 3.369557 \\ \mathrm{H} & 0.019486 & 0.391578 & 5.132393 \\ \mathrm{H} & -0.345378 & -2.525229 & 0.977565 \\ \mathrm{H} & -1.690283 & -2.656092 & 3.351817 \\ \mathrm{H} & 1.100004 & -2.894913 & 3.265408\end{array}$

$\begin{array}{rrrr}\mathbf{1 0} & & & \\ \mathrm{La} & 0.040421 & 0.162766 & 0.136996 \\ \mathrm{C} & 2.564377 & -1.082704 & -0.222082 \\ \mathrm{C} & 2.017396 & -0.958402 & -1.523746 \\ \mathrm{C} & 2.006631 & 0.419641 & -1.867308 \\ \mathrm{C} & 2.539109 & 1.145564 & -0.772951 \\ \mathrm{C} & 2.881689 & 0.217589 & 0.245008 \\ \mathrm{C} & -2.283454 & 1.597634 & -0.631030 \\ \mathrm{C} & -1.910083 & 0.853631 & -1.777614 \\ \mathrm{C} & -2.142583 & -0.520174 & -1.501339 \\ \mathrm{C} & -2.666662 & -0.621890 & -0.188332 \\ \mathrm{C} & -2.748077 & 0.686071 & 0.352586 \\ \mathrm{H} & 3.353315 & 0.455590 & 1.191627 \\ \mathrm{H} & 2.732085 & -2.009310 & 0.312792\end{array}$




$\begin{array}{rrrr}\mathrm{H} & 1.711553 & -1.776543 & -2.166174 \\ \mathrm{H} & 1.691770 & 0.837136 & -2.816726 \\ \mathrm{H} & 2.705335 & 2.217189 & -0.740823 \\ \mathrm{H} & -3.146604 & 0.947937 & 1.326091 \\ \mathrm{H} & -2.265633 & 2.678649 & -0.540501 \\ \mathrm{H} & -1.557292 & 1.263537 & -2.717045 \\ \mathrm{H} & -1.997379 & -1.341497 & -2.194149 \\ \mathrm{H} & -2.972597 & -1.535001 & 0.307152 \\ \mathrm{H} & -1.540465 & -1.849937 & 3.609124 \\ \mathrm{Sn} & -0.003980 & -1.082159 & 3.486397 \\ \mathrm{Sn} & 0.204336 & 0.446996 & 5.910237 \\ \mathrm{H} & -0.108069 & -1.619859 & 1.447849 \\ \mathrm{H} & 1.361986 & -2.129668 & 3.535242 \\ \mathrm{H} & 0.122819 & 0.489233 & 2.502683 \\ \mathrm{H} & -1.056681 & 1.627212 & 6.027139 \\ \mathrm{H} & 0.123290 & -0.509007 & 7.349282 \\ \mathrm{H} & 1.693111 & 1.327266 & 5.983070\end{array}$

12

$\begin{array}{llll}\text { C } & 0.628048 & 1.240567 & -2.650763\end{array}$

$\begin{array}{llll}\text { C } & 1.626269 & 0.354831 & -2.166088\end{array}$

$\begin{array}{llll}\text { C } & 1.100733 & -0.961907 & -2.214957\end{array}$

$\begin{array}{llll}\text { C } & -0.221009 & -0.887633 & -2.721244\end{array}$

$\begin{array}{llll}\text { C } & -0.511348 & 0.475326 & -2.993252\end{array}$

$\begin{array}{llll}\text { La } & -0.375392 & 0.327685 & -0.148116\end{array}$

$\begin{array}{llll}\text { C } & 0.218507 & -1.484395 & 2.008940\end{array}$

$\begin{array}{llll}\text { C } & 1.186109 & -0.462262 & 2.102430\end{array}$

$\begin{array}{llll}\text { C } & 0.534030 & 0.727850 & 2.529579\end{array}$

$\begin{array}{llll}\text { C } & -0.834061 & 0.442418 & 2.721483\end{array}$

$\begin{array}{llll}\text { C } & -1.039376 & -0.933631 & 2.405061\end{array}$

$\begin{array}{llll}\mathrm{H} & -2.045048 & 1.752328 & -0.072360\end{array}$

$\mathrm{H} \quad 2.247353 \quad-0.574163 \quad 1.910053$

$\begin{array}{llll}\mathrm{H} & 1.013619 & 1.681764 & 2.721057\end{array}$

H $\quad-1.587312 \quad 1.127510 \quad 3.088242$

$\begin{array}{llll}\mathrm{H} & -1.904714 & -1.521678 & 2.708995\end{array}$

$\mathrm{H} \quad 0.408348 \quad-2.523334 \quad 1.765173$

H $\quad 2.634440 \quad 0.627715 \quad-1.873532$

$\mathrm{H} \quad 1.633756 \quad-1.869407 \quad-1.953851$

$\mathrm{H} \quad-0.876040 \quad-1.728521 \quad-2.918820$

$\mathrm{H} \quad-1.433309 \quad 0.861108 \quad-3.411003$

H $\quad 0.730107 \quad 2.313544 \quad-2.771553$

$\begin{array}{llll}\text { Sn } & -3.296178 & -0.830955 & 0.440952\end{array}$

$\begin{array}{llll}\mathrm{H} & -4.228327 & -0.021479 & 1.686347\end{array}$

$\begin{array}{llll}\mathrm{H} & -4.336799 & -2.276972 & 0.376443\end{array}$

13

$\begin{array}{llll}\text { C } & 0.752004 & 1.206009 & -2.638400\end{array}$

$\begin{array}{llll}\text { C } & 1.560826 & 0.230746 & -2.001216\end{array}$ 


$\begin{array}{crrr}\mathrm{C} & 0.900384 & -1.022818 & -2.096823 \\ \mathrm{C} & -0.319548 & -0.821034 & -2.787698 \\ \mathrm{C} & -0.412814 & 0.556357 & -3.115416 \\ \mathrm{La} & -0.642139 & 0.489272 & -0.292803 \\ \mathrm{C} & 0.018754 & -1.396205 & 1.845427 \\ \mathrm{C} & 0.981713 & -0.378316 & 1.872372 \\ \mathrm{C} & 0.368581 & 0.810529 & 2.386840 \\ \mathrm{C} & -0.967876 & 0.523545 & 2.673111 \\ \mathrm{C} & -1.216147 & -0.853013 & 2.338318 \\ \mathrm{H} & -0.488050 & 2.624404 & -0.162299 \\ \mathrm{H} & 2.024902 & -0.483838 & 1.596889 \\ \mathrm{H} & 0.856227 & 1.766671 & 2.530250 \\ \mathrm{H} & -1.687966 & 1.206843 & 3.107418 \\ \mathrm{H} & -1.968622 & -1.463237 & 2.844926 \\ \mathrm{H} & 0.184459 & -2.430087 & 1.566997 \\ \mathrm{H} & 2.538117 & 0.403944 & -1.564628 \\ \mathrm{H} & 1.285116 & -1.974685 & -1.748850 \\ \mathrm{H} & -1.035522 & -1.590215 & -3.053893 \\ \mathrm{H} & -1.218281 & 1.025409 & -3.669818 \\ \mathrm{H} & 0.986481 & 2.256555 & -2.748088 \\ \mathrm{Sn} & -3.313970 & -1.175469 & 0.738508 \\ \mathrm{H} & -4.439694 & -0.737273 & 2.018109 \\ \mathrm{H} & -3.360655 & -2.911219 & 1.025940\end{array}$

\section{6}

$\begin{array}{llll}\text { Sn } & -0.786771 & 2.054210 & -0.942642\end{array}$

$\begin{array}{llll}\text { Sn } & 0.361051 & -0.972416 & 0.184622\end{array}$

$\mathrm{H} \quad-0.479874 \quad-0.280564 \quad 1.602368$

$\mathrm{H} \quad 0.597274 \quad 0.976816 \quad-0.475032$

$\mathrm{H} \quad-1.163490 \quad-0.905470 \quad-0.769763$

H $\quad 0.003666 \quad 3.573419 \quad-0.798800$

$\begin{array}{llll}\mathrm{H} & -1.140910 & 1.709379 & -2.578184\end{array}$

$\begin{array}{llll}\mathrm{H} & -2.146203 & 1.985849 & 0.081273\end{array}$

\section{5}

$\begin{array}{llll}\text { Sn } & -1.022839 & 1.877446 & -0.774716\end{array}$

$\begin{array}{llll}\text { Sn } & 0.267098 & -0.713723 & 0.167328\end{array}$

$\begin{array}{llll}\mathrm{H} & -0.127356 & 0.308636 & 1.510136\end{array}$

H $\quad 1.333586 \quad 0.167851 \quad-0.876689$

$\begin{array}{llll}\mathrm{H} & -1.195096 & -1.027052 & -0.709478\end{array}$

$\mathrm{H} \quad-0.069973 \quad 3.224246 \quad-0.275968$

$\begin{array}{llll}\mathrm{H} & -1.144956 & 1.883212 & -2.493899\end{array}$

H $\quad-2.601753 \quad 2.027479 \quad-0.100155$
18
$\begin{array}{llll}\text { C } & 2.569400 & -1.168019 & -0.566445\end{array}$
$\begin{array}{llll}\text { C } & 2.019528 & -1.060567 & -1.869786\end{array}$ 


$\begin{array}{cccc}\mathrm{C} & 1.955365 & 0.316538 & -2.206919 \\ \mathrm{C} & 2.454022 & 1.058898 & -1.106665 \\ \mathrm{C} & 2.835922 & 0.140478 & -0.093009 \\ \mathrm{La} & 0.003595 & -0.017525 & -0.209548 \\ \mathrm{C} & -2.249833 & 1.514935 & -0.962159 \\ \mathrm{C} & -1.870084 & 0.817397 & -2.136271 \\ \mathrm{C} & -2.147903 & -0.560181 & -1.935173 \\ \mathrm{C} & -2.709531 & -0.709666 & -0.641263 \\ \mathrm{C} & -2.769910 & 0.571191 & -0.037672 \\ \mathrm{Sn} & -0.035477 & -0.869066 & 2.904313 \\ \mathrm{H} & 3.291238 & 0.391048 & 0.857633 \\ \mathrm{H} & 2.774309 & -2.089291 & -0.034410 \\ \mathrm{H} & 1.749828 & -1.887302 & -2.517536 \\ \mathrm{H} & 1.624396 & 0.724960 & -3.154646 \\ \mathrm{H} & 2.578110 & 2.136035 & -1.070063 \\ \mathrm{H} & -3.181949 & 0.794229 & 0.939099 \\ \mathrm{H} & -2.202361 & 2.588985 & -0.816787 \\ \mathrm{H} & -1.479482 & 1.261985 & -3.044338 \\ \mathrm{H} & -2.010925 & -1.349567 & -2.665843 \\ \mathrm{H} & -3.057298 & -1.635922 & -0.199490 \\ \mathrm{H} & -1.423776 & -1.549590 & 3.729403 \\ \mathrm{H} & -0.169879 & -1.901119 & 1.300147 \\ \mathrm{H} & 1.236558 & -1.804096 & 3.666018 \\ \mathrm{H} & 0.212043 & 0.765733 & 5.618292 \\ \mathrm{Sn} & 0.312144 & 0.879258 & 7.332993 \\ \mathrm{H} & -1.033021 & 1.772123 & 7.919709 \\ \mathrm{H} & 0.307215 & -0.691880 & 8.022242 \\ \mathrm{H} & 1.763923 & 1.692422 & 7.760240\end{array}$

$\begin{array}{cccc}\mathbf{1 7} & & & \\ \mathrm{C} & 2.447535 & -1.259111 & -0.516394 \\ \mathrm{C} & 1.908323 & -0.879448 & -1.771212 \\ \mathrm{C} & 2.013835 & 0.532690 & -1.886909 \\ \mathrm{C} & 2.609938 & 1.023931 & -0.699115 \\ \mathrm{C} & 2.874948 & -0.082246 & 0.150300 \\ \mathrm{La} & 0.042513 & 0.106137 & 0.063698 \\ \mathrm{C} & -2.330207 & 1.484126 & -0.632687 \\ \mathrm{C} & -1.891394 & 0.858377 & -1.825732 \\ \mathrm{C} & -2.054766 & -0.544198 & -1.668785 \\ \mathrm{C} & -2.603209 & -0.781878 & -0.383425 \\ \mathrm{C} & -2.766992 & 0.471023 & 0.260838 \\ \mathrm{Sn} & 0.002668 & -0.929135 & 3.290138 \\ \mathrm{H} & 3.370940 & -0.042098 & 1.113267 \\ \mathrm{H} & 2.541583 & -2.271185 & -0.142855 \\ \mathrm{H} & 1.533544 & -1.553052 & -2.533367 \\ \mathrm{H} & 1.733850 & 1.122789 & -2.751975 \\ \mathrm{H} & 2.865051 & 2.058738 & -0.496115 \\ \mathrm{H} & -3.205345 & 0.630127 & 1.239473 \\ \mathrm{H} & -2.374725 & 2.553293 & -0.453965\end{array}$




$\begin{array}{rrrr}\mathrm{H} & -1.538921 & 1.362204 & -2.718375 \\ \mathrm{H} & -1.848822 & -1.296076 & -2.422034 \\ \mathrm{H} & -2.873321 & -1.747570 & 0.025395 \\ \mathrm{H} & -1.489144 & -1.479693 & 3.911897 \\ \mathrm{H} & -0.109099 & -1.747894 & 1.346736 \\ \mathrm{H} & 1.402064 & -1.728125 & 3.855108 \\ \mathrm{H} & 0.157299 & 0.785586 & 3.642961 \\ \mathrm{Sn} & 0.202265 & 0.667133 & 6.153056 \\ \mathrm{H} & -1.092872 & 1.795008 & 6.426168 \\ \mathrm{H} & 0.157022 & -0.437586 & 7.496484 \\ \mathrm{H} & 1.664833 & 1.587336 & 6.349500\end{array}$

